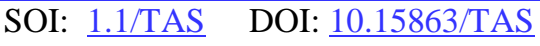 International Scientific Journal Theoretical \& Applied Science}

p-ISSN: 2308-4944 (print) ｅ-ISSN: 2409-0085 (online)

Year: $2015 \quad$ Issue: 03 Volume: 23

Published: $30.03 .2015 \quad$ http://T-Science.org
Sergey Aleksandrovich Vorobyev

Candidate of Technical Sciences, associate professor of Don State Technical University, Russia v.serge.79@mail.ru

Sergey Sergeevich Vorobyev

Candidate of Technical Sciences, associate professor of Don State Technical University, Russia

Andrey Stanislavovich Reshenkin

Candidate of Technical Sciences, head of department of Don State Technical University, Russia

Roman Aleksandrovich Goncharov

Candidate of Technical Sciences, associate professor of Don State Technical University, Russia

Alexander Grigoryevich Tihomirov

Candidate of Technical Sciences. associate professor of Don State Technical University, Russia

\title{
THE DYNAMIC CHARACTERISTICS OF VEHICLE SUSPENSION SYSTEMS WITH OPTIMAL DAMPING
}

Abstract: Provides research in the field of effective systems of protection suspension vehicles undergoing dynamic influence of road surface irregularities. The results allow to determine the further development of the theory of optimal control in damping vibrations.

Key words: dynamics, vehicle suspension, damping, oscillations, optimality.

Language: Russian

Citation: Vorobyev SA, Vorobyev SS, Reshenkin AS, Goncharov RA, Tihomirov AG (2015) THE DYNAMIC CHARACTERISTICS OF VEHICLE SUSPENSION SYSTEMS WITH OPTIMAL DAMPING. ISJ Theoretical \& Applied Science 03 (23): 93-96.

Soi: http://s-o-i.org/1.1/TAS*03(23)17 Doi: crossef http://dx.doi.org/10.15863/TAS.2015.03.23.17

\section{ДИНАМИЧЕСКИЕ ХАРАКТЕРИСТИКИ ПОДВЕСКИ АВТОМОБИЛЯ С СИСТЕМАМИ ОПТИМАЛЬНОГО ДЕМПФИРОВАНИЯ КОЛЕБАНИЙ}

Аннотация: Приведены исследования в области разработки эффективных систем защиты подвесок автомобилей, подвергающихся динамическому воздействию неровностей дорожного покрытия. Результаты исследований позволяют определить дальнейтее развитие теории оптимального управления демпфированием колебаний.

Ключевые слова: динамика, подвеска автомобиля, демпфирование, колебания, оптимальность.

В настоящее время ведущие мировые автопроизводители предлагают системы подвесок с улучшенными характеристиками, например с возможностью автоматической адаптации к различным дорожным условиям (активные или адаптивные подвески), подвески, позволяющие изменять дорожные просвет (гидравлические, пневматические, винтовые) [1]. Однако те или иные виды «комфортных» подвесок остаются доступными либо в определенном сегменте транспортных средств, либо у конкретных автопроизводителей или разработчиков. Для большинства же серийных автомобилей проблема оптимальной конструкции подвески остается актуальной.

Весьма актуальна защита оборудования и людей от качки, тряски, вибраций и ударов при движении транспортных средств по неровностям дороги, особенно в условиях, когда время на транспортировку ограничено [2-4].

В статье предлагается методика оценки динамических характеристик подвески с системами оптимального демпфирования колебаний. Методика предназначена для расчёта вертикально-угловых колебаний двухосного транспортного средства при движении по неровной дороге (рис. 1).

В этом случае использование систем оптимального демпфирования достаточно эффективно [5-8] Для исследований была принята расчетная схема транспортного средства с двухточечным опиранием (рис.2). В качестве обобщенных координат были выбраны перемещение точек над опорами $\mathrm{z}_{1}, \mathrm{z}_{2}$.

Математическая модель оптимального демпфирования колебаний в этом случае имеет вид: 
$M_{1} \ddot{z}_{1}+M_{3} \ddot{z}_{2}+k_{1} z_{1}+c_{1} z_{1}=k_{1} \dot{q}_{1}(t)+c_{1} q_{1}(t)$
$M_{3} \ddot{z}_{1}+M_{2} \ddot{z}_{2}+k_{2} z_{2}+c_{2} z_{2}=k_{2} \dot{q}_{2}(t)+c_{2} q_{2}(t)$

$t=0, \quad z_{1}=z_{2}=\dot{z}_{1}=\dot{z}_{2}=0 ;$
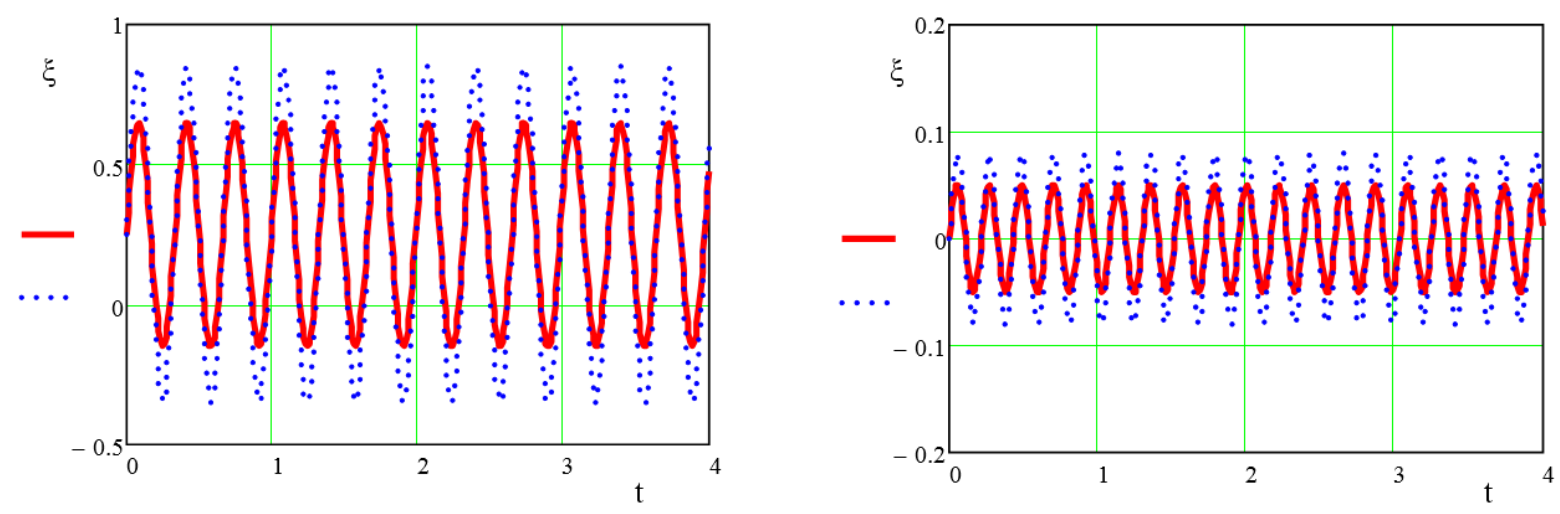

Рисунок 1 - Вертикально-угловые колебания двухосного транспортного средства при движении по неровной дороге: а) передней оси б) задней оси.

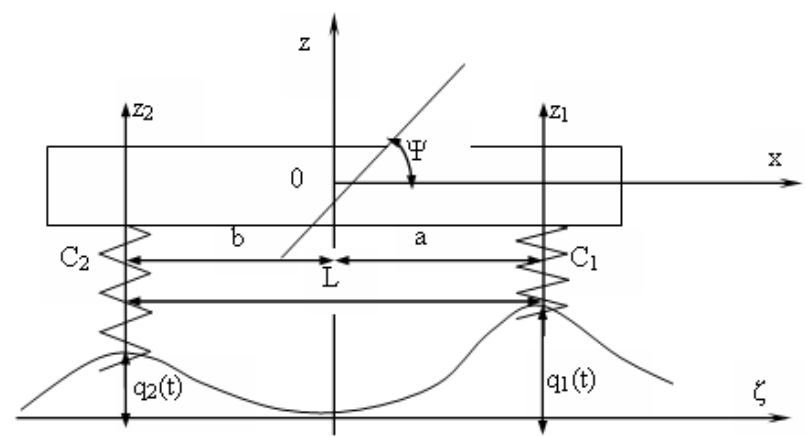

Рисунок 2 - Модель объекта с двумя точками подвеса.

$$
c_{j}=\left\{\begin{array}{cc}
c_{j \max }, & \dot{z}_{j}\left(z_{j}-q_{j}\right)>0 ; \\
c_{j \min }, & \dot{z}_{j}\left(z_{j}-q_{j}\right) \leq 0 ;
\end{array}\right.
$$

где $M_{1}, M_{2}$ - приведенные к осям массы; $M_{3}$ масса инерционной связи; $q_{j}(t)-$ профиль дорожной неровности, $k_{1}, k_{2}$ коэффициенты вязкого сопротивления.

Сравнительные результаты расчетов для случаев оптимального и неоптимального демпфирования показаны на рис.3, 4. Пунктиром показан процесс, идущий без управления, сплошной линией - c управлением, показана также функция управления. Во всех случаях подтверждается высокая эффективность оптимальных систем.

При определении эффективности гашения колебаний при оптимальных постоянных параметрах в качестве исходной была принята расчетная схема вертикальных колебаний контейнера в пусковой установке. Движение такой системы представлено математической моделью с одной степенью свободы с кинематическим возбуждением колебаний:

$$
\begin{aligned}
& m \ddot{z}+k \dot{z}+c z=c q(t)+k \dot{q}(t), \\
& t=0, \quad z=z_{(0)}, \quad \dot{z}=\dot{z}_{(0)}, \\
& q(t)=q_{0} \text { Sinvt. }
\end{aligned}
$$

Здесь $v$ - частота внешнего кинематического воздействия.

$$
\eta=\frac{z_{1}}{q_{1}}
$$

Оценка эффективности гашения колебаний осуществлялась по критерию точности:

$$
J=\frac{1}{2} \int_{0}^{t_{K}}(z-y)^{2} d t
$$

где $y(t)$ - желаемое движение, определяемое для случая оптимального демпфирования колебаний.

$$
m \ddot{y}+\tilde{c} y=\tilde{c} q(t)
$$




$$
\begin{gathered}
\tilde{c}= \begin{cases}c_{\text {max }}, & \dot{y}(y-q)>0 ; \\
c_{\text {min }}, & \dot{y}(y-q) \leq 0 ;\end{cases} \\
t=0 ; \quad y=z_{0} ; \quad \dot{y}=\dot{z}_{0}
\end{gathered}
$$$$
\dot{p}_{1}=u_{1} ; \quad \dot{\lambda}_{1}=-(z-y) \Gamma_{1} ;
$$

$$
\dot{p}_{2}=u_{2} ; \quad \lambda_{2}=-(z-y) \Gamma_{2} ;
$$$$
m \ddot{\Gamma}_{1}+k \dot{\Gamma}_{1}+c \Gamma_{1}+z=q_{0} \sin v t ;
$$

Оптимизации подлежали коэффициенты жесткости и сопротивления $p_{1}=c, \quad p_{2}=k$. Дифференциальные уравнения для параметров и функций чувствительности имеют вид:

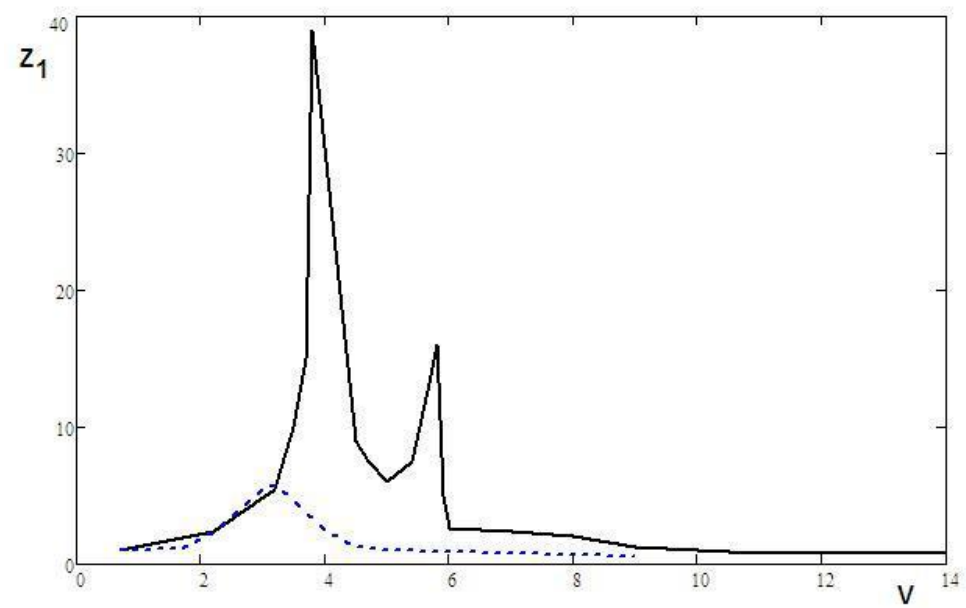

Рисунок 3 - Зависимость коэффициента динамичности от частоты внешнего воздействия для передней оси.

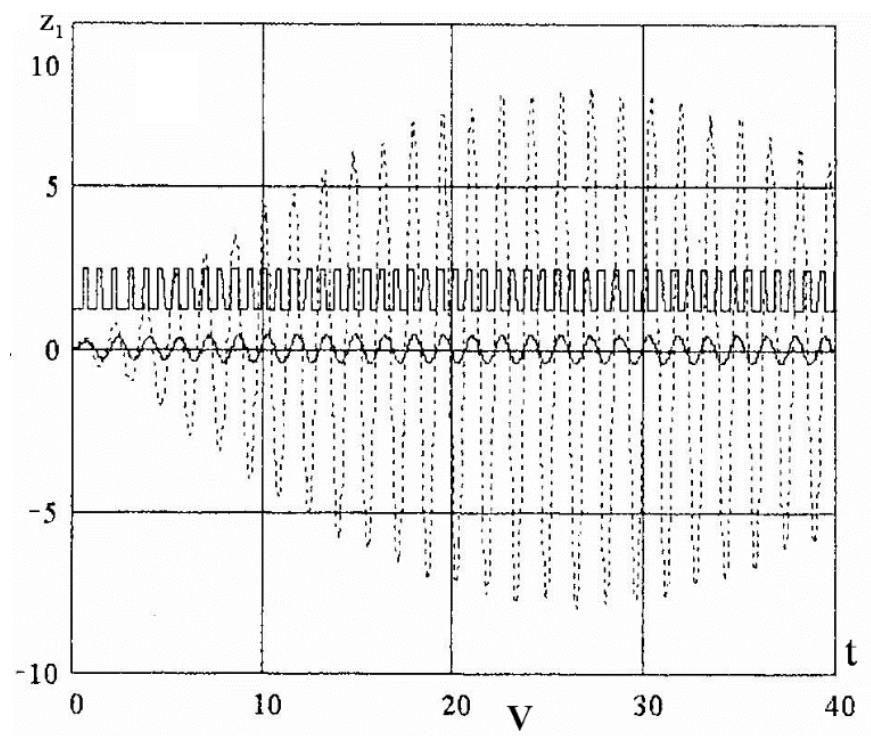

Рисунок 4 - Зависимость коэффициента динамичности от частоты внешнего воздействия задней оси.

Характерные графики колебаний представлены на рис.5.
Приведенные в статье исследования находятся в рамках разработки эффективных систем защиты подвесок, подвергающихся динамическому

ISPC The Best of European Innovations, 
воздействию неровностей дорожного покрытия. Получила дальнейшее развитие теория оптимального управления демпфированием колебаний, методы численного решения оптимизационных задач, предложены перспективные решения, которые могут быть использованы в современной автоиндустрии.
Эти результаты могут быть полезны также специалистам других направлений, связанных как с приложениями теории оптимального демпфирования колебаний, так и к приложению теории оптимального управления другими процессами [9-10].

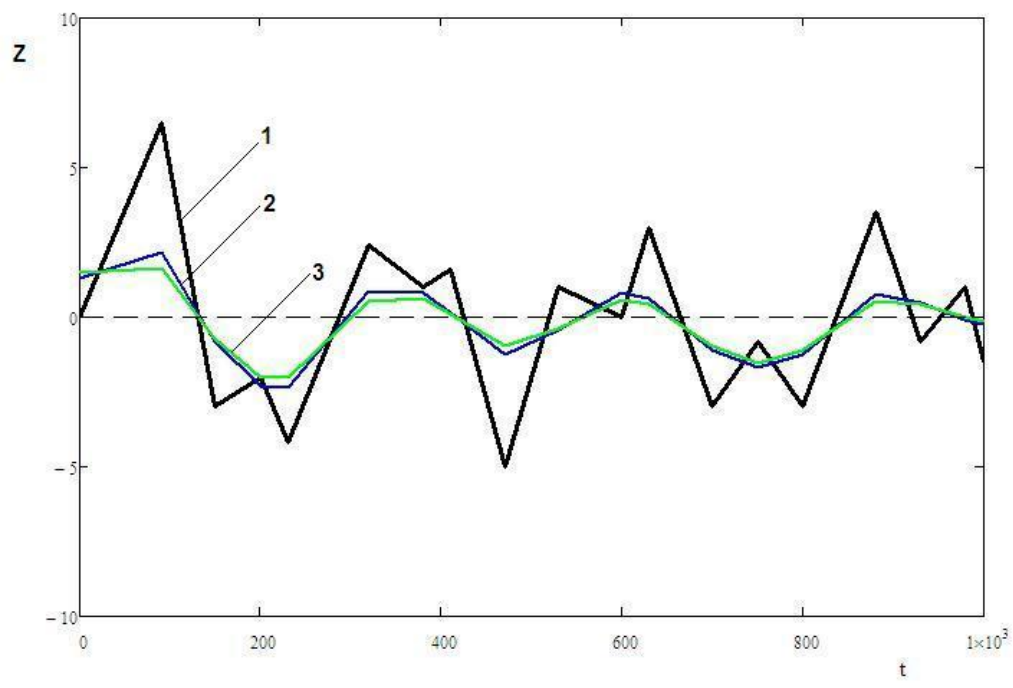

Рисунок 5 - Графики колебаний: 1- колебания без демпфирования; 2- колебания при оптимальном демпфировании; 3 - колебания при оптимальном выборе $c, k$.

\section{References:}

1. Kuchvid RP (2001) Ispitaniya avtomobilya:uchebnikMoscow, MGIU, 2001. -351 p.

2. Kostoglotov AI, Shevcova LA (1987) Optimalnoe vozbuzhdenie rezonansnykhkolebanij uprugikh system prikladnaya mekhanika, 1987, T.23 №6, p.23-30.

3. Kovaleva AS (1990) Upravlenie kolebatelnymi i vibroudarnymi sistemami. Moscow, -Nauka1990. $-256 \mathrm{p}$.

4. Natshif A, Dzhouns D, Khenderson D (1988) Dempfirovanie kolebanij. Moscow, -Mir. 1988. $-448 p$.

5. Bishop R (1986) Kolebaniya /per s angl. pod red.-Ya.G Panovko.-3-e-izd, Moscow, Nauka1986. $-190 \mathrm{p}$.

6. Komkov V (1975) Teoriya optimalnogo upravleniya dempfirovaniem kolebanij prostykh-uprugikh sistem, -Moscow, -Mir1975. -158p.

7. Varava VI (1986) Prikladnaya teoriya amortizacii transportnykh mashin - L.: Izd-vo LGU-1986. -188 p.

8. Butkovskij AG (1975) Metody upravleniya sistemami s raspredelennymi parametrami, Moscow, -Nauka, 1975. -568 p.

9. Vorobyev SA, Kostoglotov AI, Kulechov AV (2000) Mgnovenno-optimalnoe dempfirovanie kolebanyi mnogomassovych system c uprugimi svyazyami.-Izv.Vuzov, Sev-Kav. Region, Estesstvennye nauki, 2000, №2. pp. 29-32.

10. Vorobyev SA, Kostoglotov AI, Kulechov AV (2001) Sposob dempfirovaniya kolebanyi uprugopodvechennogo obyekta.. - Patent PF na izobreteniee №2162034 ot 20.01.01. 